\title{
The best task allocation process is to decide on one's own: effects of the allocation agent in human-robot interaction on perceived work characteristics and satisfaction
}

\author{
Alina Tausch ${ }^{1}$. Annette Kluge ${ }^{2}$ (I)
}

Received: 21 November 2019 / Accepted: 16 November 2020 / Published online: 21 December 2020

(c) The Author(s) 2020

\begin{abstract}
New technologies are ever evolving and have the power to change human work for the better or the worse depending on the implementation. For human-robot interaction (HRI), it is decisive how humans and robots will share tasks and who will be in charge for decisions on task allocation. The aim of this online experiment was to examine the influence of different decision agents on the perception of a task allocation process in HRI. We assume that inclusion of the worker in the allocation will create more perceived work resources and will lead to more satisfaction with the allocation and the work results than a decision made by another agent. To test these hypotheses, we used a fictional production scenario where tasks were allocated to the participant and a robot. The allocation decision was either made by the robot, by an organizational unit, or by the participants themselves. We then looked for differences between those conditions. Our sample consisted of 151 people. In multiple ANOVAs, we could show that satisfaction with the allocation process, the solution, and with the result of the work process was higher in the condition where participants themselves were given agency in the allocation process compared to the other two. Those participants also experienced more task identity and autonomy. This has implications for the design of allocation processes: The inclusion of workers in task allocation can play a crucial role in leveraging the acceptance of HRI and in designing humane work systems in Industry 4.0.
\end{abstract}

Keywords Task allocation $\cdot$ Decision-making $\cdot$ Autonomy $\cdot$ Task identity $\cdot$ Human-robot interaction (HRI) $\cdot$ Trust

\section{Introduction}

Industry 4.0 stands for a plethora of new technologies, interconnected processes, and innovative ways of producing goods. These developments do not only offer many opportunities for efficiency and flexibility, but also for designing humane and future-proof work. The use of, for example, human-robot interaction (HRI) in smaller scale production settings can change the way tasks are distributed and thereby the responsibility a worker has for the production process and their own work design. A future assembly

Alina Tausch

Tausch.alina@baua.bund.de

1 Scientific Management Products and Work Systems, Federal Institute for Occupational Safety and Health, Dortmund, Germany

2 Chair of Work and Organisational Psychology, Ruhr University Bochum, Bochum, Germany situation might look like this: Each worker is responsible for the entire production process of a particular product from start to finish. The worker has their own collaborative robot that they can work with throughout the production process. As the robot is highly flexible, it can be used for different tasks and can work in cooperation or in close collaboration, handling work pieces together with the worker. The worker is in charge of structuring the work process and allocating the various task segments either to themselves or to the robot. This arrangement gives the worker ownership over the whole (segment of the) production process and enables him to react to changes in production requirements quickly and autonomously.

Even in Industry 4.0 settings, where many processes might function fully autonomous, people will still be needed for certain tasks. Those people need to be kept "in the loop" to understand the processes, to react to changes flexibly, and to solve problems dynamically. A task allocation in HRI that is influenceable for the workers allows for combining the advantages of human creativity, flexibility and 
problem-solving abilities with the efficiency, accuracy, and speed of the robot. When such synergies are created through dynamic task allocation, production is ready for the challenges of tomorrow's working world.

\subsection{Task allocation in human-robot interaction}

Task allocation is a vitally important process in every production task and especially in designing human-robot interaction. It is the decisive preparation step shaping the task characteristics and the cooperation of humans and machines. Over $70 \%$ of a sample of manufacturers and system integrators stated that it is a large or very large challenge for the broader implementation of HRI (Gaede et al. 2018). It is defined as the distribution of tasks between humans and machines as a part of work organization (Older et al. 1997). From a traditional perspective, it is a design process done by a specialist team that considers the subtasks and the pros and cons an execution by either a human or a machine would have (Stanton et al. 2005). More recent approaches tend towards more flexibility by using adaptive algorithms able to reschedule (see, e.g., Nikolakis et al. 2018).

Abdallah and Lesser (2005) subdivide the allocation process into several stages: subdivision of one major task in various subtasks, the definition of the appropriate agent for execution, and the negotiation of task execution between the agents. For a more detailed consideration, the process can be further divided. Abdallah's and Lesser's structure would be part of (1) the decision-making process itself, followed by (2) the allocation resulting from it, (for example, see Michalos et al. 2018) and (3) the execution of subtasks as to the allocation solution. The last element is (4) the completion of the full task, as conceptualized in the task regulation theory by Hacker and Sachse (2014).

Research on allocation has been ongoing for a long time, with publications, e.g., from Sheridan (1984) reporting on experiments about supervisory control involving allocating tasks to support systems. Several researchers from different fields have endeavored to find the "optimal" allocation result, from early approaches by Fitts (1951) to more recent articles, e.g., by Michalos et al. (2018). They consider distributing the subtasks to humans and machines, oftentimes robots, so as to maximize efficiency or speed, sometimes also taking into account workload (Debernard et al. 1993) or acceptability (Alami et al. 2006). The result of these studies is always some kind of an optimizing algorithm that finds the "best" solution for task distribution to humans and robots in relation to the chosen goal criteria.

However, this approach only solves part of the problem of finding a sensible allocation solution. What these studies do not look at is the human side of cooperation with a robot. Studies like that of Gombolay et al. (2015), who look at shared agency between human and robots in task allocation and resulting satisfaction, are still quite rare and do not study large samples. Nevertheless, Gombolay's study hints at people preferring decision-making by the system or with system involvement, possibly because productivity is better in these conditions. It also shows that people prefer a cooperating system as a team partner and feeling more important to the task in a shared decision (Gombolay et al. 2015). Munzer et al. (2017) show that users prefer a robot that acts semi-autonomously and initiates actions on its own compared to one that needs to be assigned each task manually. The reasons for these findings require further research. It can at least be questioned whether workers indeed prefer "automatic" allocation-as the conditions in the studies presented led to a more fluent and more efficient task execution when autonomous allocation takes place.

What is currently missing is a human-centered perspective specifically on allocation processes. Several authors such as Fantini et al. (2017) looking at including operators in changing robotic programs, Habib et al. (2017) considering human needs and abilities in controlling swarms of robots, or Romero et al. (2015) proposing a reference architecture to engineer automation systems that are human-centered have already addressed the need for taking a human-centered view in Industry 4.0. Now, more research is needed that goes even deeper into the topic of task allocation design to find out how the allocation decision can be made to create an allocation result and a work process that do not lead to overload or underload and that are satisficing to human needs. This is of vital importance, especially in human-robot interaction that relies on the willingness to cooperate (for an examination of the relations between willingness to cooperate and variables such as system use or trust, see Rajaonah et al. 2008) as well as on human abilities and on the willingness to execute tasks. As to Rücker et al. (2018), human-robot interaction is mostly looked at from a technical perspective, whereas the involvement of humans is, from a socio-technical perspective, of vital importance. Joint optimization (Trist 1981), i.e., designing the social and the technical system together, is key to well-functioning socio-technical systems. In a recent article on control in human-machine interaction, Flemisch et al. (2019) look at the history of the concepts of shared control and cooperation and underline the importance of these constructs for designing successful human-machine systems. If the human worker is not considered, he or she cannot or does not cooperate adequately with the technology. Consequences of this can be found in research on the ironies of automation (for an overview, see Onnasch et al. 2014): Overreliance on automated functioning can, e.g., lead to a loss in perceived control and in the ability to handle unexpected situations. This danger is not new, but of regrowing importance when Industry 4.0 visions become reality in highly complex production settings with cyber-physical systems steering production processes. That is why this study seeks to show how 
the needs of the workers can and should be considered in the design of allocation processes.

\subsection{The role of the allocation agent}

The main interest of this paper centers on a part of the allocation process that offers new possibilities as technology and organizations evolve: the allocation agent. Interactive software and hand-guided teaching of collaborative robots allow workers to have an influence on the allocation process, while the development of algorithms and artificial intelligence introduces computer systems as new decision agents.

In production, task allocation is normally a preparatory task (Hacker and Sachse 2014) carried out by a productionplanning unit that consists of experts for process design [see also Stanton et al. (2005)]. However, as production develops and more dynamic processes with more individualized products in smaller batch sizes evolve, the ex-ante planning of tasks might not be practicable anymore. An alternative to this is adaptive automation, i.e., the dynamic allocation of control over functions of the system to either a person or a machine for performance optimization (Kaber and Riley 1999). Research on this can be found, e.g., with Cosenzo et al. (2010) comparing teleoperation, semi-autonomy, and adaptivity of a robot. Hoc and Lemoine (1998) in their experiments differentiate allocation done by the human together with a system, a system alone, or another person with system support. These different viewpoints show that task allocation can generally be made by (1) a centralized organizational unit, (2) a (possibly intelligent) automated system, or (3) a person involved in production. The latter could be the person directly working with the robot or a further qualified machine manager on the shop floor. Allocation could also be executed by a form of interaction of the different agents (as seen in Hoc and Lemoine 1998), as the prominent levels of automation concept (Parasuraman et al. 2000) suggests: it resolves the question who-man or machine-should take over a function in a system by formulating different degrees of system influence over a decision.

Allocation by a centralized unit is the dominant form of allocation in practice, and is mostly researched under the topic of expert planning, for example in the aviation context in Ryan et al. (2014). Here, experts often work together with some kind of automation, the so-called decision support systems. This term is rather an umbrella term for computers involved in a decision-making process (Keen 1980). Nevertheless, Keen (1980) goes on to emphasize the key role of the interaction of human and system: "The label 'Support System' is meaningful only in situations where the 'final' system must emerge through an adaptive process of design and usage" (p. 28). This shows the meaning of the human as a user of the system.
Fully automated allocation is researched for example by Tsarouchi et al. (2017). Alami et al. (2005) present a framing model for task allocation executed by a robot in a human-robot interaction: Here, the supervision kernel of the robot selects and defines tasks and initiates execution. The researchers call for a robot task planning that is reasonable, socially accepted, and understandable for the human in addition to being safe and executable (Alami et al. 2005).

About task allocation done by people on the shop floor, not much is known from practical application, as a production-planning unit is still the standard in the industry. One can draw insights from research on participation and work design, which reveals, for example, the importance of autonomy for motivation and health (Hackman and Oldham 1975). We also see some more current research focusing more on the role of the human in influencing the autonomy mode of a robot (Baker and Yanco 2004) and in meta-controlling robotic autonomy (Goodrich et al. 2001) or in an allocation decision (Gombolay et al. 2015). The samples used for such research, however, are small and the research field is only in its infancy.

Therefore, this paper seeks to investigate the effects of these three different task allocation agents on perceived work characteristics and satisfaction by comparing different allocation scenarios. Generally, we assume that the involvement of the worker in task allocation leads to positive effects on their experience of the allocation as well as on perceived work resources. These assumed effects are further detailed in hypotheses 1-5 in the following sections. Sub-hypotheses with an additional numeral (e.g., 3.1) describe additional influence factors on these effects or on the outcomes addressed in the main hypotheses.

\subsection{The impact of task allocation on perceived work characteristics}

Task allocation has a significant impact on how work characteristics are perceived. It determines the overview over the full task as well as how free one feels in fulfilling their own tasks. Autonomy, also referred to as (job) control, is a task characteristic describing how much freedom an individual worker has regarding their work schedule, making decisions, and the methods applied (Morgeson and Humphrey 2006). It is a vitally important resource in work. Wieland and Hammes (2014) point out that especially controllability of one's own work situation leads to control experience, which is in turn decisive for work outcomes such as health and performance. One possible way to create controllability is to involve people in decision-making processes.

Hypothesis 1: The experimental group allowed to allocate tasks themselves (worker condition) perceives more task autonomy than each of the groups with 
another agent making the decision (robot and management condition).

Task identity is a characteristic describing a task as a cohesive unit with distinct results (Sims et al. 1976). The assembly of a product from start to finish is high in task identity, while only screwing one part to another with the product then moving on-without the person knowing what happened to the product before and what happens nextis very low in task identity. Hackman and Oldham (1980) assert that tasks high in identity are more interesting, and the JD-R model (Bakker and Demerouti 2017) also considers task identity as a resource. Hacker and Sachse (2014) define "complete" tasks as those that fulfill the following five characteristics: they offer sufficient task demands, so that they do not result in inactivity; they make cooperation possible, give opportunities for own or cooperative goal-setting and decision-making, and combine cognitive with productive elements; and they trigger learning. We assume that having people participate in the planning and allocation of tasks contributes to the experience of task identity.

Hypothesis 2: The experimental group allowed to allocate tasks themselves perceives higher task identity than each of the groups with another agent making the decision (robot and management-determined allocation).

\subsection{The impact of task allocation on satisfaction with process, allocation, and work results}

For the assessment of the allocation process, it is important how the decision process and its results as well as the indirect impact on the work result are perceived. A rather general measure for the assessment of those factors is the satisfaction with each of them. As Zhang and Fitzsimons (1999) state, the affective experience of decision processes can be shaped by the decision situations' characteristics. In this study, we therefore hypothesize that satisfaction with the individual process steps is influenced especially by the allocation agent that is manipulated, as well as a number of influence factors explained in the following.

\subsubsection{Satisfaction with the allocation process and mediating influences}

Process satisfaction is a variable largely overlooked in research, though studies like that by Zhang and Fitzsimons (1999) still show that it is a variable distinct from decision result satisfaction [see operationalization by Fitzsimons et al. (1997)] that is affected by the design of decision processes.

In this study, we expect the allocation process satisfaction to be high when the person to whom tasks are allocated is involved in the decision-making process. For example, a study from market research has shown that greater participation in the use of recommendation agents for online shopping is connected to greater satisfaction (Dabholkar and Sheng 2012). Locke et al. (1986) claim that employees wish to have influence in decisions that have an impact on their work, and that such influence can be reached through participation or delegation. This goes along with the assumptions of control theories like the theory of cognized control (Frey and Jonas 2002), stating that the amount of experienced control results from explainability, predictability, and influenceability of events-in decision situations where one is involved, decisions are more explainable and predictable, and certainly influenceable. Therefore, we assume that an allocation situation where the worker has influence will be more satisficing than a decision made by someone else without opportunity for participation or self-determination.

Hypothesis 3: When the workers are allowed to allocate tasks themselves (i.e. in the worker-condition), the satisfaction with the allocation process is highest.

We also assume that there are multiple factors in addition to the allocation agent itself that influence satisfaction with the process. As a meta-analysis about control at work shows, autonomy is related to work satisfaction and shows similar patterns as participation (Spector 1986). Hence, the effect of the agent on process satisfaction might be partially explainable by the autonomy one gains through being able to decide on the allocation (see hypothesis 1).

Hypothesis 3.1: Perceived autonomy mediates the influence of the allocation agent on process satisfaction.

In cases where a business unit or a system decides on the allocation, autonomy should be equally low (as long as the worker is not involved in their decisions), so that additional factors related to the decision basis and the decision agent should be relevant for process satisfaction. On the one hand, this can be the experience of the criteria used to make the decision. Criteria appropriateness is the subjective rating that someone else has based their decision on the "right" and relevant decision criteria. From the perspective of task regulation theory (Hacker and Sachse 2014), this rating is a result of the comparison of own goals and the criteria used for the judgement. Although not really considered in psychological studies on decision perception, the authors view subjective appropriateness to be influential in the experience of allocation process satisfaction.

On the other hand, trust is an important influence factor connected to the agent responsible for the decision. Moray et al. (2000) underline the importance to understand the psychological interactions between trust, the feeling of self-confidence, and the interaction of human and machines to understand how adaptive automation can work. 
A meta-analysis by Hancock et al. (2011) analyzes trust in human-robot interaction and comes to the conclusion that characteristics of the robot are most influential for trust in HRI. They cite, among other research, a study by Freedy et al. (2007), who claim that trust is especially important in HRI as it influences the willingness to accept information and suggestions given by a robot. This view is supported by Inagaki and Itoh (2013), who differentiate between overtrust and overreliance in their theoretical framework, with the first being a psychological state founded in a diagnosis of an object (inappropriately) as trustworthy and the latter being an action selection process that can be influenced by trust. Thielsch et al. (2018) show that in situations at work where information systems are trusted, wellbeing is rated higher. As Driscoll (1978) shows, the satisfaction with organizational decision-making is best predicted by organizational trust, which underlines the additional importance of agent perception for a satisfaction judgement.

Hypothesis 3.2: Perceived criteria appropriateness and trust in the allocation agent have a positive influence on the satisfaction with the allocation process when the decision is made by another agent.

\subsubsection{Satisfaction with the allocation decision and the mediating role of task characteristics}

The design of the allocation process should not only have an effect on the perception of the process itself, but also on the perception of the allocation solution and the resulting performance. Allocation (solution) satisfaction is, similarly to decision satisfaction, the amount of satisfaction with the outcome choice (Zhang and Fitzsimons 1999). As one can influence the distribution of tasks, the solution is more likely to express one's needs and preferences. From research on job crafting, we know that job crafting behavior predicts need satisfaction, which then predicts wellbeing of employees (Slemp and Vella-Brodrick 2013). The same should hold for the more specific task allocation behavior, which allows employees to choose the tasks they would like to do and avoid those tasks that are stressful, straining, or boring to them.

Hypothesis 4: In the group in which tasks are allocated by the workers, the satisfaction with the allocation result is higher than when another agent makes the decision.

This might be due to the work design that results from the allocation decision. A certain division of labor leaves the job with certain characteristics. For example, task identity is affected by which subtasks one executes-if the subtasks are preparatory as well as executional, task identity is higher in comparison to a job where one is assigned only different preparation subtasks. Also autonomy is highly influenced by task allocation, not only autonomy in deciding about the allocation, but also in executing the tasks that are allocated to the human. The satisfaction with the resulting distribution should be explainable by how the work a person is left with after the distribution is designed and which resources it offers.

Hypothesis 4.1: Task characteristics, i.e., autonomy and task identity, explain parts of the variance in solution satisfaction.

\subsubsection{Satisfaction with the result of work and the mediating role of goal attainment}

We assume that the effects of the allocation agent reach even beyond the allocation process and the resulting allocation. It should also affect the satisfaction with what has been reached with the work process, meaning the outcomes of work.

Hypothesis 5: In the group in which tasks are allocated by the workers themselves, group members are more satisfied with the result of the work that has been executed with the robot.

From the viewpoint of task regulation theory (Hacker and Sachse 2014), each work assignment is reinterpreted to a personal task. This reinterpretation process is influenced by individual beliefs and goals that shape what one thinks is necessary and what is to be achieved. When it comes to finishing a task, the results are measured against these personal goals (Hacker and Sachse 2014). If the worker has been given the possibility to bring their personal beliefs into the process of task distribution, it is more likely that these goals will be satisfied and that, in turn, the worker will be satisfied with the result.

Hypothesis 5.1: The relationship between the influence over the allocation and satisfaction with the work result is mediated by how much workers can reach their own goals for a task.

\subsubsection{Differences between decision agents}

Until this point, we have mainly considered the differences between an allocation decision that the person affected is involved in and a decision made by someone else (a business unit or a robot), because we expect to find the highest effects in these contrasting conditions. Not less interesting is the question of differences in the satisfaction compared between decisions made by management and those made by machines, in this case robots. 
Research question: To what extent does satisfaction with the allocation process, its allocation and work results differ in situations where the decision-maker is either a robot or a management unit?

\subsection{Overview over the hypotheses}

The hypotheses looked at in this study consider the effect of the allocation agent in an HRI setup on...

- The task characteristics autonomy (H1) and task identity (H2);

- The satisfaction with the allocation process itself (H3);

- The satisfaction with the allocation resulting from the process (H4);

- The satisfaction with the work results after working with the robot as allocated (H5).

We also included sub-hypotheses describing the assumed mechanisms behind these effects and additional influences on the outcomes:

- Mediation of the effect on process satisfaction via autonomy (H3.1);

- Additional influence on process satisfaction of criteria appropriateness and trust in the case of an allocation without the worker involved (H3.2);

- Additional influence on allocation solution satisfaction of autonomy and task identity (H4.1);

- Mediation of the effect on work result satisfaction via personal goal attainment (H5.1).

\section{Methods}

\subsection{Sample}

The participants were acquired using personal networks as well as online platforms such as Thesius or SurveyCircle. All participants were German speaking, as the questionnaire was fully in German. One hundred and seventyeight people completed the questionnaire without being offered a gratification. Of those, 27 were excluded from the analyses, because they did not pass the control items or the manipulation check.

After this, 151 participants remained, who were assigned to the three experimental conditions as follows: 49 were in the organization-allocation condition, 52 in the robot-allocation condition, and 50 in the worker-allocation condition. As multiple ANOVAs reveal, the subgroups did not differ in gender, age, education, or working status.

One hundred and one of the participants were female, two categorized themselves as "diverse" and 28 were male. The average age was $28.85(\mathrm{SD}=0.72)$. The sample consisted of 81 students, 52 employees, one employee articled, three civil servants, six self-employed, and six people doing else (with 117 participants working in total). Nineteen of the participants stated to have experience in working with a robot.

\subsection{Experimental design}

The design is an experimental between-subjects design with three groups that are compared on a number of outcome variables. The participants were randomly assigned to one of the three experimental conditions via a function integrated in the online questionnaire. After about half of the participants were acquired, we calculated the group sizes after dropouts and corrected the group sizes that served as a basis for randomized assignment within the online questionnaire. We used the website http://www. soscisurvey.de to generate the questionnaire and conduct the experiment. The whole study lasted from January 2019 to March 2019. During this time, the experiment was available online and accessible for people interested in the study.

The online experiment was conducted combining a framing story, an experimental manipulation to the scenario (see Sect. 2.1), and questionnaires investigating the psychological consequences of the scenario presented (see Sect. 2.3). The structure of the online experiment can be found in Fig. 1. The average processing time for the whole experiment was $696.84 \mathrm{~s}$ (=11.61 $\mathrm{min}), \mathrm{SD}=212.85$, with a slightly longer processing time in the worker-allocation condition, $M=736.34, \mathrm{SD}=215.25$.

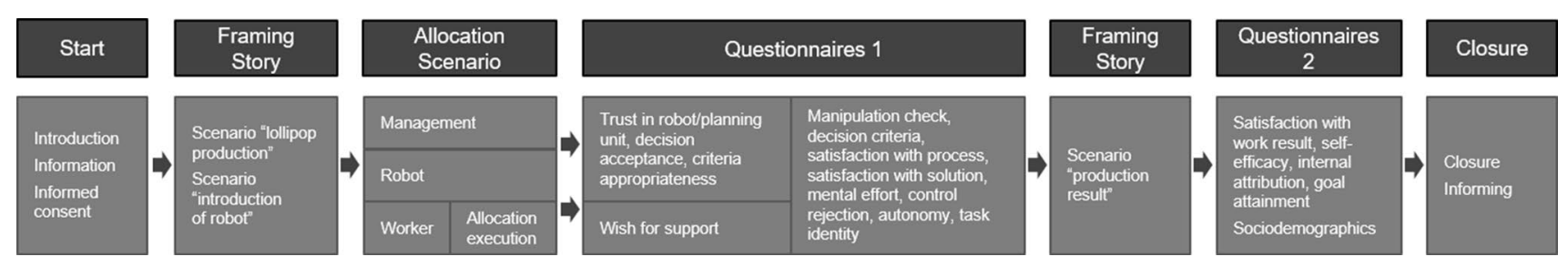

Fig. 1 Structure of the online experiment 


\subsection{Scenario and experimental manipulation}

At first, the study participants were introduced into the scenario that they later needed to refer to in the questionnaires. They were told to imagine being a worker at the "Sugarshock Corp", a production plant for sweets, and responsible for the production of lollipops. The production process, consisting of eight distinct steps that need to be followed one after another, was explained with one sentence for each step and shown in pictures (see Fig. 2). The production steps were described as follows (translation from the German description that could lead to slight differences in the understanding):

1. At first, a box of lollipop sticks has to be collected from the warehouse.

2. These sticks then have to be positioned in the holding device 100 pieces at a time.

3. The lollipop mass is mixed from a combination of sugar, water, flavors, and coloring.

4. Just at the right point in time, when the mass is not too hot anymore, it is poured into the molds.

5. Now, a sizzling sugar ball has to be pressed in each mold.

6. The sticks are placed into the lollipop heads.

7. After drying, the lollipops are loosened from the molds by jiggling mildly.

8. In the end, the lollipops are equipped with a wrapping paper. The Sizzling Lollies are done.

The participants were then told that a robot has been provided to support them at their workplace. It was said to be able to do the same production steps as the human worker, with the exception of one task. Their form of cooperation is therefore designed as being augmentative (based on the classification by Schmidt 1994), with both of them having (nearly) the same abilities and combining their workforces to augment their capacity to solve the production task. In the scenario, human and robot work together cooperatively, meaning that they share working time, workspace, and an aim, i.e., producing the lollipops. In contrast to this, there is also collaboration, meaning that, in addition to a shared aim, there is direct contact between human and robot, which is not the case in this scenario as subtasks are not shared (for the classification, see Schmidtler et al. 2015).

The description of the robot was kept short and neutral not to influence participants into an either very positive or very negative expectation towards working with it. In the following, participants were allocated to one of the three experimental conditions without their knowledge and were then presented the matching scenario of task allocation.

The participants were lead through the scenario by a "foreman" via a text block and told that the allocation of tasks would be made by the robot, by a management unit or by the workers themselves (see Fig. 2). In condition 1 and 2 , the task allocation was prescribed and presented to the participants as being a management decision $(1$, in the latter referred to as management condition) or the decision of the robot (2, referred to as robot condition). The resulting allocation of tasks was the same, with human and robot each having four tasks allocated to them. Pictures supported the impression of either a manager or the robot deciding about the tasks.

In condition 3 (worker condition), the participants were deciding on the allocation themselves. They were not given additional information but just a selection of tasks. Now, they could decide which of the tasks they wanted to do themselves and which tasks were to be allocated to the robot. There was no production goals given in any conditions. The allocation by the worker was not restricted (neither in time nor in selectability of tasks): it was only given that step 5 of the production process (pressing the sizzling balls into the sugar mass) could not be done by the robot. This was done to omit participants allocating all tasks to the robot and to
Robot-Condition

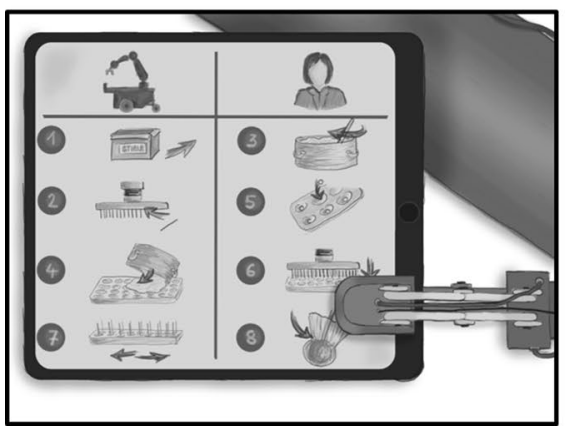

Management-Condition

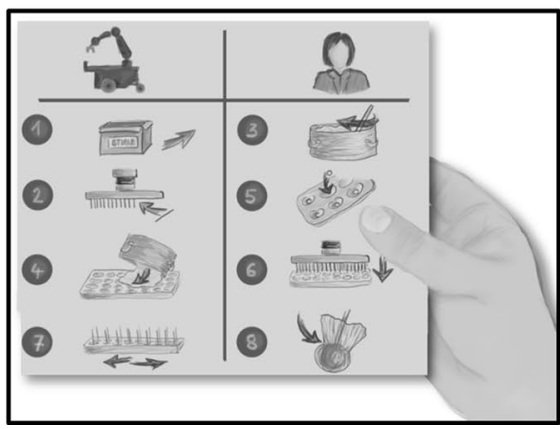

Worker-Condition

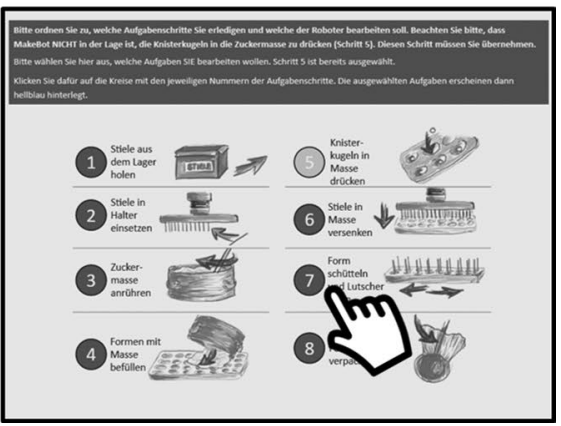

Fig. 2 Presentation of the allocation result in the three experimental conditions. Note: The third part of the figure is a screenshot of the online questionnaire- the text on there is in German explaining the participants how to allocate the tasks and naming the different production steps 
add realism to the scenario, as mostly robots are not suitable for executing all tasks a human can, especially not those involving fine motor skills.

The subsequent questionnaires addressed the experience of the allocation decision and the resulting work. After the first block of questionnaires, the participants were confronted with the last situation of the scenario: their workday is over and a short note tells them the production amount they reached throughout the day. It was the same amount of 10,000 lollypops in each condition, without any reference figures throughout the experiment or other information on how "good" or "bad" this production result might be. This last part of the scenario was followed by questions concerning this result of work and sociodemographic questions.

\subsection{Independent and dependent variables}

The aim of this study is to explore the influence the allocation agent has on different subjective outcome variables and how these effects are mediated. Therefore, we manipulated the independent variable, the allocation agent, by setting up three different experimental conditions: It is either the robot, a management unit, or the participant. The manipulation happens via the different scenario descriptions and the associated pictures presented to the participants within the study. Each participant only experiences one variant of the independent variable.

As dependent variables, we looked at task characteristics on one hand and satisfaction on the other hand. The task characteristics which we considered most important were autonomy, i.e., freedom in methods, planning and decisionmaking, and task identity. With regard to satisfaction, we looked at three different forms. First, we are interested in satisfaction with the allocation process itself, meaning with the way the decision is made. This is differentiated from satisfaction with the allocation decision, which refers to the resulting division of labor. Finally, we looked at satisfaction with the result of work, i.e., how satisfied people were with what has been achieved by the task execution of them and the robot.

Three additional variables that are assumed to function as mediators between the independent variable and the dependent ones were also considered: (1) the attainment of personal goals, referring to how good people were able to achieve what they wanted with the cooperative task; (2) perceived appropriateness of the criteria on which the allocation decision was based; and (3) trust in the decision agent, that is in the robot or in the organizational unit. Variables 2 and 3 were only relevant for the scenarios in which the allocation agent was either the robot or the management unit.

All variables and their connection assumed in the hypotheses, are depicted in Fig. 3.

\subsection{The applied questionnaire instruments}

Beyond the scales given in Table 1, there were a few nominal questions included: a control item demanded to tick the rightmost box to check for proper reading of the

\section{Independent Variable}

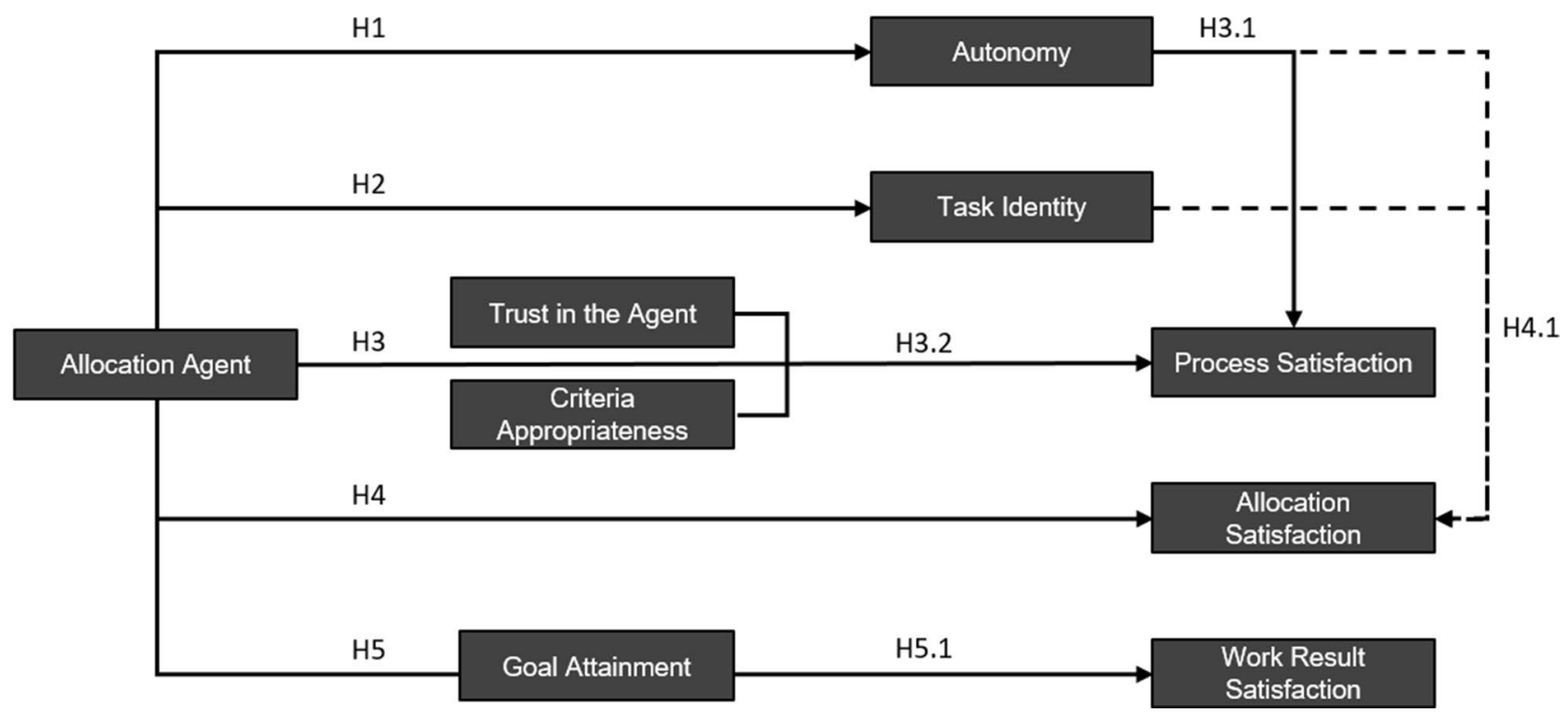

Fig. 3 Independent and dependent variables and their assumed connections 
Table 1 Scales used in the experiment

\begin{tabular}{|c|c|c|c|c|c|}
\hline Construct & Source & Example item & Items & Scale & $\alpha$ \\
\hline Autonomy & Stegmann et al. (2010) & $\begin{array}{l}\text { The job gives me considerable opportunity for independ- } \\
\text { ence and freedom in how I do the work }\end{array}$ & 9 & $1-5$ & 0.94 \\
\hline Task identity & Stegmann et al. (2010) & $\begin{array}{l}\text { The job is arranged, so that I can do an entire piece of } \\
\text { work from beginning to end }\end{array}$ & 4 & $1-5$ & 0.87 \\
\hline Satisfaction with... & Newly developed items & & & & \\
\hline Allocation process & & $\begin{array}{l}\text { I am this satisfied with the way the allocation decision has } \\
\text { been made }\end{array}$ & 4 & $1-6$ & 0.84 \\
\hline Allocation decision & & $\begin{array}{l}\text { I am this satisfied with how the tasks are allotted to me } \\
\text { and the robot }\end{array}$ & 3 & $1-6$ & 0.98 \\
\hline Result of work & & I am this satisfied with the result of our work & 3 & $1-6$ & 0.96 \\
\hline Goal attainment & Newly developed items & I could reach my goals for this working day & 3 & $1-5$ & 0.91 \\
\hline Criteria appropriateness & Newly developed item & The decision criteria probably applied are appropriate & 1 & $1-5$ & l \\
\hline Trust in automation/management & Jian et al. (2000) & The robot/organizational unit is trustworthy & 12 & $1-7$ & $0.93 / 0.86$ \\
\hline
\end{tabular}

The example items here are translations of the German items used. Slight differences in wording or meaning can occur due to language differences. The items for autonomy and task identity are taken from the original-language questionnaire, reported by Stegmann et al. (2010)

questions. The manipulation check asked participants to rate whether the allocation decision has been made by the management, the robot, or themselves. All of the participants were asked what kind of decision criteria they used for allocating the task or what they think the management/robot has considered, respectively (e.g., sequence of steps, efficiency, and how complex or difficult the task are).

As scales for autonomy and task identity, we opted for the nine- and four-item measurements from the Work Design Questionnaire (Morgeson and Humphrey 2006), as it is a well-established questionnaire for research on work design and is also available in a validated German version (Stegmann et al. 2010). The trust scale taken from Jian et al. (2000) was chosen, because it was developed empirically and considers trust and distrust (based on empirical evidence) as two opposites on one dimension with no need to use two different scales. The scale is aimed at evaluating trust between humans and automated systems, but has proven to be suitable for measuring trust between humans as well (Jian et al. 2000). It was used with only slight changes in the wording to adapt to either the management or the robot as the object of trust. The other scales or items were developed newly for this study, as there were no suitable measurements available.

In cases where we needed to calculate with a steady variable instead of a categorical one (as given by the experimental conditions), we used the measurement of the subjective influence participants had on the allocation decision. They were asked to rate their influence on a scale from one (none) to five (full).

\subsection{Exclusion criteria}

Before analyzing the data, we needed to select the relevant data by excluding incomplete or poor-quality questionnaires. As the experiment was conducted online with no preselection of participants, these steps were necessary to obtain valid data.

The first step in data preparation was the selection of only those questionnaires that have been completed until the last page, independent of individual missing data entries during the questionnaire. The next step was to exclude all cases in which participants stated to not have answered the questionnaire properly and ask not to use their data for analyses (filter 1 ; nine excluded). All participants who did not answer the control item correctly were also filtered out (filter 2 ; nine excluded).

People who answered the manipulation check with the "I don't know" option were excluded (four excluded). We excluded all those who answered the manipulation check ("Who has allocated the tasks?") wrong, as well (five excluded). An exception to this was the combination where the participant was in the robot-allocation condition and stated that the organization has allocated the task-which would be realistic.

\section{Results}

The means and standard deviations of all measured constructs can be found in Table 2. It includes also the means under the three experimental conditions that are partially tested for significant differences in the following. 
Table 2 Means and standard deviations of the measured constructs in the experimental conditions

\begin{tabular}{|c|c|c|c|c|c|}
\hline & \multirow[t]{2}{*}{ Scale } & \multirow[t]{2}{*}{$M(\mathrm{SD})$} & \multicolumn{3}{|l|}{$M(\mathrm{SD})$} \\
\hline & & & $\begin{array}{l}\text { Allocation } \\
\text { through manage- } \\
\text { ment }\end{array}$ & $\begin{array}{l}\text { Allocation } \\
\text { through robot }\end{array}$ & $\begin{array}{l}\text { Allocation } \\
\text { through } \\
\text { worker }\end{array}$ \\
\hline Satisfaction with & $1-6$ & & & & \\
\hline Allocation process & & $4.03(1.13)$ & $3.63(1.15)$ & $3.77(1.05)$ & $4.70(0.88)$ \\
\hline Allocation decision & & $4.36(1.23)$ & $3.97(1.13)$ & $4.02(1.40)$ & $5.10(0.69)$ \\
\hline Result of work & & $4.64(1.05)$ & $4.30(1.05)$ & $4.52(1.12)$ & $5.12(0.80)$ \\
\hline Appropriateness of criteria & $1-5$ & $3.71(1.05)$ & $3.00(1.00)$ & $4.00(1.00)$ & I \\
\hline Autonomy & $1-5$ & $1.99(0.95)$ & $1.46(0.51)$ & $1.59(0.69)$ & $2.93(0.81)$ \\
\hline Task identity & $1-5$ & $3.49(1.15)$ & $3.20(1.15)$ & $3.05(1.09)$ & $4.34(0.81)$ \\
\hline Personal goal attainment & $1-5$ & $3.81(0.83)$ & $3.65(0.74)$ & $3.58(0.90)$ & $4.21(0.68)$ \\
\hline Trust in automation/management & $1-7$ & $4.76(0.98)$ & $4.55(1.06)$ & $3.95(0.87)$ & I \\
\hline
\end{tabular}

When looking at the mean values of those participants stating to have experience in working with a robot and those who do not have such experience, no larger differences show, so that results are not reported here. A $t$ test only yielded a significant result for individual goal attainment, $t(149)=-2.65, p=0.009$. As the group of people experienced with robots only has a size of 19 (vs. 132), there is no way to make accurate statements about differences, and hence, they are not considered further in this study.

The correlations between the measured constructs can be found in Table 3.

The three facets of satisfaction are strongly correlated, as can be seen in Table 3, but not fully overlapping. A mediation model shows a direct effect of process satisfaction on work result satisfaction of $0.26,95 \%$ CI $[0.10,0.42]$ as well as an indirect effect via satisfaction with the allocation solution of $0.19,95 \%$ CI [0.07, 0.34]. The full model is $R^{2}=0.36, F(1,149)=85.55, p<0.001$. An approach differentiating between satisfactions with different parts of the allocation process is therefore legitimate and conducted in the following.

\subsection{Manipulation check}

Participants were asked to rate the influence they had over the allocation decision on a scale from one (none) to five (full): in the robot condition, the mean was $1.25(\mathrm{SD}=0.65)$; in the management condition, $1.27(\mathrm{SD}=0.73)$, whereas in the worker condition, the mean was $4.32(\mathrm{SD}=0.51)$, which shows a significant difference, $F(2,148)=385.72, p<0.001$. The effect size of the experimental condition on the perceived influence is large with $\eta^{2}=0.84$. This shows that the experimental manipulation has worked out well.

\subsection{Influence of the allocation agent on perceived work characteristics: autonomy and identity}

Hypothesis 1 is concerned with the difference in perceived autonomy depending on the allocation agent. Because homogeneity of variances is not given in case of autonomy, the groups were compared in a Welch test. The groups show pronounced differences in their experienced autonomy, $F(2$, $95.53)=61.81, p<0.001$, with the highest value of $M=2.93$

Table 3 Correlation coefficients of the measured constructs

\begin{tabular}{|c|c|c|c|c|c|c|c|c|c|}
\hline & & \multicolumn{8}{|c|}{$r_{s}$} \\
\hline & & 1 & 2 & 3 & 4 & 5 & 6 & 7 & 8 \\
\hline 1 & Process satisfaction & 1 & $0.62 * * *$ & $0.50 * * *$ & $0.58 * * *$ & $0.38 * * *$ & $0.31 * * *$ & $0.51 * * *$ & $0.57 * * *$ \\
\hline 2 & Decision satisfaction & & 1 & $0.57 * * *$ & $0.44 * * *$ & $0.42 * * *$ & $0.34 * * *$ & $0.57 * * *$ & $0.44 * * *$ \\
\hline 3 & Work result satisfaction & & & 1 & $0.43 * * *$ & $0.28 * * *$ & $0.43 * * *$ & $0.69 * * *$ & $0.52 * * *$ \\
\hline 4 & Criteria appropriateness & & & & 1 & $0.24 * *$ & -0.04 & $0.43 * * *$ & $0.58 * * *$ \\
\hline 5 & Autonomy & & & & & 1 & $0.34 * * *$ & $0.41 * * *$ & 0.15 \\
\hline 6 & Task identity & & & & & & 1 & $0.45 * * *$ & $0.20 *$ \\
\hline 7 & Personal goal attainment & & & & & & & 1 & $0.55 * * *$ \\
\hline 8 & Trust in automation/management & & & & & & & & 1 \\
\hline
\end{tabular}

All correlations are tested with a Spearman-test robust to violations of normal distribution. The significance of the correlations was tested using a one-sided approach, $* p \leq 0.05, * * p \leq 0.01, * * * p \leq 0.001$ 
$(\mathrm{SD}=0.81)$ in the worker-condition. This condition differs significantly $(p<0.001)$ from the management-allocation scenario $(\mathrm{MD}=1.47)$ and the robot-allocation scenario $(\mathrm{MD}=1.34)$ in a Games-Howell post hoc test. Hypothesis 1 is thus corroborated.

Differences in task identity were tested with a Welch test, $F(2,95.96)=24.93, p<0.001$. A Games-Howell test reveals highly significant mean differences of 1.04 between the worker and the management condition and of 1.19 between the worker and the robot condition. This confirms Hypothesis 2, which asserts that people with influence over the allocation perceive more task identity.

\subsection{Satisfaction with the allocation process steps}

Hypothesis 3 states that satisfaction with the decision-making process is higher when people can allocate the tasks themselves. The experimental condition has a significant effect of $\eta^{2}=0.18$ on the satisfaction with the decision process, $F(2,148)=15.74, p<0.001$. A Gabriel post hoc test reveals significant $(p<0.001)$ differences between the worker- and the robot-condition $(\mathrm{MD}=0.93)$ as well as between the worker and the management condition $(\mathrm{MD}=1.06)$. The influence on process satisfaction is partially mediated by experienced autonomy, $R^{2}=0.18, F(2$, $148)=16.03, p<0.001$, with an indirect effect of autonomy of $0.20,95 \%$ CI $[0.05,0.38]$ and a remaining direct effect of $0.34,95 \%$ CI [0.07, 0.60] (bootstrap sample of 5000). Hypothesis 3 can be maintained as well as the mediation hypothesis 3.1 .

For hypothesis 3.2, we looked at possible influence factors on satisfaction with the allocation process when the worker is not involved in the decision. Satisfaction with the decision-making process in the two conditions without influence of the participant on the allocation is explained by a combination of trust in the decision agent, $\beta=0.36$, and rated appropriateness of criteria, $\beta=0.41$, in a multiple stepwise regression, $R^{2}=0.48, F(2,98)=45.92, p<0.001$. Hypothesis 3.2 can thus be accepted.

The satisfaction with the resulting allocation solution differs between the experimental conditions, as a Welch test shows, $F(2,90.76)=24.34, p<0.001$. A Games-Howell post hoc test reveals that the differences lie between the worker and the robot condition $(\mathrm{MD}=1.08)$ and the worker and the management condition (MD $=1.13$ ), $p<0.001$. Hypotheses 4 can thus be accepted.

If one includes autonomy and task identity in the prediction of satisfaction with the allocation solution, both account for $19 \%$ of the variance, $R^{2}=0.19, F(2,148)=17.67$, $p<0.001$, with a standardized coefficient of $\beta=0.34$ for autonomy and $\beta=0.19$ for task identity. Hypothesis 4.1 , which assumes that task characteristics explain part of the variance in solution satisfaction, can thus be accepted, as well.

The satisfaction with the work result also differs between the conditions, $F(2,148)=8.92, p<0.001$. In a linear regression, the amount of influence accounts for $8 \%$ of the variance in result satisfaction, $R^{2}=0.08, F(1,149)=13.15, p<0.001$. A Gabriel's post hoc test shows significant mean differences between the worker and the robot condition (MD $=0.60$, $p=0.009)$ and the worker and the management condition $(\mathrm{MD}=0.82, p<0.001)$. Hypothesis 5 , which states that people in the condition where the worker is the allocation agent will be more satisfied with the work result, can thus be accepted.

Result satisfaction is also partially explained by the attainment of personal goals, $R^{2}=0.40, F(1,149)=98.49$, $p<0.001$. We then calculated a mediation model with influence on the allocation decision (instead of the categorical condition variable) influencing the mediator achievement of personal goals and by this work result satisfaction. This yielded a significant indirect effect of $0.21,95 \%$ CI [0.10, 0.33 ] with a bootstrap sample of 5000 , as well as a still significant direct effect of $0.20,95 \%$ CI $[0.03,0.36]$ and a total effect of $0.41,95 \%$ CI $[0.21,0.61]$. The prediction model for work result satisfaction is $R^{2}=0.42, F(2,148)=53.41$, $p<0.001,1$ with a coefficient of the $b$-path of 0.20 and one of the $c^{\prime}$-path of 0.75 . Hypothesis 5.1, which postulates that personal goal achievement acts as a mediator between influence over the allocation and the amount of satisfaction with the work result, can thus be confirmed.

The analysis presented above is sufficient to answer the research question regarding the differences in satisfaction between the robot and management-allocation condition. Neither process, nor decision or work result satisfaction revealed any differences between the two scenarios. The descriptive statistics in Table 2 do not show any remarkable differences, and the post hoc tests only yielded significant results between the worker and the other two conditions. We can answer the question if there are differences in the outcome variables between an allocation by the management and an allocation by a robot with a "no" in this case.

\subsection{Allocation choices in the worker condition}

In the condition where participants decided themselves on the task allocation, they chose on average 2.46 tasks $(\mathrm{SD}=1.54)$ for themselves (in addition to the default task of pressing sizzling balls into the mass of sugar). Six of the participants chose no tasks for themselves and one person chose all tasks. In total, there were 37 different allocation patterns chosen, with no identifiable preference for one specific pattern, except for the one with no tasks for the worker. The task chosen most often (by 24 of the 50 participants) was to steer the sugar mass, followed by collecting the box 
of lollipop sticks (22) and filling the sugar into the form (21). The least chosen was to prepare the sticks by putting them in the holder (12).

\section{Discussion}

The objective of the study was to examine the impact the decision agent has on the perception of a task allocation process in HRI. We were able to show that it makes a big difference for satisfaction as well as for work design who decides over task allocation. When the participants were allowed to decide on their own about task allocation between them and the robot, they perceived more task identity and more autonomy in their (imaginary) work task. They were also more satisfied with the allocation process, which is partially mediated by autonomy. In the conditions with no worker influence on the allocation decision, i.e., when the robot or the management allocated the tasks, process satisfaction is explained by trust in the decision agent and appropriateness of perceived decision criteria. A person deciding on their own is also more satisfied with the resulting allocation solution, which is again explained by the task characteristics autonomy and task identity. Those people are also more satisfied with the work result at the end of the working day. This is due to a better attainment of personal goals with the result of work. There are no significant differences between the allocation by the management and the allocation performed by the robot. The three satisfaction facets are dependent of one another with a higher process satisfaction leading to more allocation solution satisfaction and, directly and indirectly, to a higher satisfaction with the result achieved by the working process.

The experience of more task identity when one is responsible for allocating tasks arises from being confronted with the task of structuring, thinking about the subtasks and who should execute them. This triggers a deeper understanding of the task as a whole and its execution process. Hervé et al. (2000) write that task identity comes from being responsible for a whole piece of work with a distinct result, which can be reached, e.g., by a distribution of duties. When a person is responsible for the allocation process, they are responsible for the whole task. Therefore, they can experience more task identity compared to when they are only assigned a set of subtasks, but do not necessarily know or have not thought about the whole execution process. Autonomy at work, here seen as an amalgamation of planning, decision-making, and method autonomy, as well profits from having the power to decide over the task allocation to oneself and a robot. Being autonomous in this one decision and the choice of workforce for the different tasks has a cascading effect on the whole work experience. This can be due to the vast implications the task allocation decision has for the work of the person affected-it basically shapes their working day-or to the importance of the decision. If a person is allowed to make such an important decision as planning their own work and that of the robot, it might be that they feel autonomous overall. The mechanism can be that of a halo effect, with one dominant aspect of, in this case the work situation, influencing the overall impression of autonomy at work.

Although participants were only imagining working with a robot in a production task, we can see strong effects of the involvement in the allocation decision on both allocation process and solution satisfaction. We know from literature from diverse fields how important participation can be: e.g., Bucknall and Thomas (1996) could show that the involvement of nurses in critical everyday decisions correlates with task satisfaction. Delegating allocation decisions can also be motivational for the agent, as management literature argues (Van den Steen 2006). From psychology, we know about the effects decision latitude has on lowering mental strain (Rystedt et al. 2007) or on satisfaction (Cheung et al. 2015) in manual work, but not much about the influence of task allocation and its specific effects. The known effects are in line with the result found here, that influence on allocation determines autonomy, which then has the known effects on satisfaction. In literature about decision satisfaction, Lantz et al. (2005) could show that when the individual preference for participation is matched, people are more satisfied with decision-making processes and solutions. Therefore, maybe, it is not a matter of "more is better" when it comes to influence, but a fit between individual needs and opportunities that a work offers for those needs to be satisfied. This needs further investigation in the allocation context in future studies.

Especially interesting is the huge influence of decisionmaking authority on the perception of the work result (for a discussion on the concepts of authority, responsibility, and control, see Flemisch et al. 2012). The result was only presented to the participants as a number of lollipops they produced, with no reference value and no difference in the number between the conditions. Therefore, it is quite striking that still participants perceived this number differently depending on whether they were or were not in charge of allocating tasks. The effects that can be expected in reality, when the worker really executes a task together with a robot and is left with a real production output in the end of the day, might be even larger. The pride elicited by a feeling of success leads to perseverance in following tasks, even if they require more effort and are not enjoyable, as, for example, demonstrated by Williams and DeSteno (2008). This shows the relevance a positive perception of work results has for work behavior of employees. Especially, manufacturing work is often characterized by repetitive and sometimes straining tasks. There, this feeling of accomplishment might be of vital importance to maintain motivation and engagement at 
work. It is important to consider that the employees also set themselves goals, e.g., to produce a certain amount or realize a zero-failure process, which they can be proud of when they are able to realize them. If this positive perception of the work result and the achievement of personal goals can be supported by involving people in allocating their own tasks, this can be a tool worth considering in motivation of employees.

The strong connections between satisfaction with the decision process, decision outcome, and work results are not surprising. In a publication on decision justice, Brockner and Wiesenfeld (1996) argue that peoples' reactions to their environment (and that includes decision processes at work) are determined by a combination of procedural and distributive influencing factors. Transferred to our findings, this underlines the interaction between decision processes and solutions when it comes to their perception and evaluation by the people involved. As well as this, a common-method bias might contribute to the results. In all three cases, participants were asked, in multiple items with different wording, how satisfied they were with either the process, the decision, or the result. We tried to minimize this by measuring the three facets at different points of time in the questionnaire and thus keeping them separate in the presentation. That results are still similar can be expected and is deliberate, as different facets of the same construct, namely satisfaction, are measured. As they follow the process of task allocation, which leads from an allocation decision process to an allocation solution, and then to a work result after execution, it is only logical that satisfaction measurements for the distinct steps build upon one another. If one is satisfied with a decision process, it is also more likely that they will be satisfied with the decision resulting from it and with what is reached through that allocation in the end. Nevertheless, we were able to show that allocation process, solution, and work result satisfaction are distinct facets and that one can work on each of them specifically.

We could not find significant differences between the perception of the robot allocation and the allocation made by the company's management. This might be because both are external decisions and people only differentiate between those decisions where they are involved or in charge and those others make. In addition to this, both "external" agents in this experiment were rather impersonal: the participants had no personal connection to the decision system in the robot or the planning unit. The results of Hoc and Lemoine (1998) show that people accept an allocation more when it was made by another human knowing their and the support system's activities than when it was made just by the system. This suggests that there might be different assessments in "real" allocation situations than in this online experiment and that the participants might just not have been able to differentiate between the agents. In the manipulation check, we asked the participants who made the allocation decision. In the robot-allocation condition, about $40 \%$ of the participants stated that the company (not the robot) has made the allocation decision. Another explanation of this could be that participants see the responsibility for the allocation with the company programming the decision system implemented in the robot and therefore perceive the resulting allocation process and result the same.

Two different factors that were found to make a substantial difference in the perception of the allocation made by another agent- though the agent itself is not in this studyare trust and criteria appropriateness. If a person considers the basis of the decision appropriate, i.e. reasonable, and if the agent deciding is deemed trustworthy, it seems irrelevant if it is a machine or a human being. Although the irrelevancy is to be regarded with caution (see above), the finding on the importance on appropriateness and trust holds massive implications for system design as well as for transparency and communication.

Looking at the pattern of allocated tasks in the condition with free choice, there were no naturally preferred allocation solutions. A small amount of people agreed on allocating everything possible to the robot, probably for efficiency reasons, but other patterns did not show as being preferred and the choice of tasks did not show an effect on the satisfaction variables. This is probably due to the whole process being an artificially created scenario that participants did not really need to work in, so their choice did not have an actual effect on their duties. We would expect different results in a real-world allocation task and address this topic in the limitations section.

\subsection{Limitations}

Our findings and the conclusions are subject to some limitations due to the study design and sample. The sample was in a smaller age range and mainly female, which might be considered as not representative for the working population in all production settings. Nevertheless, we managed to recruit mainly people who were employed and by this had an idea about how it is to have tasks allocated to oneself. Of course, it would have been interesting to test production workers and people who have already been confronted to working with a robot, but we did not have a field access and so were dependent on voluntary participation. As the study is easy to conduct once set up, we would highly encourage other researchers to use our design on a more practically relevant sample.

A similar problem results from the design of the study: we used an online experiment to work around not being able to conduct a field study where we could investigate the impact of real-life allocation situations. The imaginary scenario allowed us to access the field of human-robot interaction, 
which is not yet widespread in the industry and thus is hard to evaluate in a practical context. This design unfortunately limits generalizability of the results, as no real allocation happened, and the allocation decision did not have real consequences for the work of the participants. A meta-analysis on the effect of fair explanations for decisions by Shaw et al. (2003) that included an analysis of the study results with different methodologies found the correlations in vignette studies, i.e., studies using stories as a reference for studying people's perceptions and attitudes (Hughes 1998), to be the same as in non-vignette ones. That speaks for the ability of studies such as ours to gain insights into a research topic, despite the methodological limitations and the difference to a realistic laboratory experiment or even a field study.

Nevertheless, a study with a vignette presenting a simplified situation cannot represent the complexity of a real production or a real task planning process containing workflow planning, idle times, etc. We cannot say how much the participants' answers were influenced by their ideas on these factors we did not manipulate or address (e.g., how much their assessment of the amount of output was influenced by prerequisite interferences). In addition to this, we only looked at subjective outcome variables and did not include more objective measurements like real production results or measures of the interaction quality, which would hold further insights into the complex consequences of task allocation in HRI. Another aspect linked to complexity is not included in our simplified scenario: the dynamic of Industry 4.0-scenarios in general and of task allocation approaches that suit these new demands in special.

Nevertheless, this online study is a first approach to the topic and will be further developed into a laboratory experiment by the authors. We are currently working on a setup with different allocation situations followed by a cooperative task participants execute together with a robot to gain more insights and validate our findings in a more realistic setting.

\subsection{Practical implications}

Work designers can take from this study that whenever an external agent is in control of an allocation decision, it is important to use acceptable criteria for the allocation and to have a decision agent that is trustworthy, independent of it being an automated system like a robot or a business unit. Both of those can be reached by designing good communication with the employees, by transparent information on criteria and processes and an opportunity for feedback. Those can be offered both by a human and by a decision system. However, this experiment could only investigate a limited range of decision agents and this only in a fictional setting. It is probable that, in reality, it is still relevant who makes a decision, especially if it is a clearly recognizable person the worker has a personal connection to. There are probably interaction effects between the agent and their trustworthiness: a certain person might be preferred as a decision-maker if they are a respected leader, but there might also be preference for a more neutral automated decision system if it is considered fairer.

The most important practical implication of this study is that the design of task allocation processes is something worth considering. Until today, much energy has been invested in finding good allocation solutions, whereby the decision-making process also has manifold effects on employees. These first results imply that, whenever possible, authority over task allocation should be given to the employee involved in task execution. This leverages satisfaction and feeling of autonomy as well as the achievement of personal goals in work. In a small scale, rather unstandardized production settings, this is conceivable. An employee could be given control over a task and a robot and could then autonomously decide on the best way to execute the production task. This could be supported by a joint setup of decision criteria that there are to apply when allocating tasks. Especially, the use of collaborative robots with easier programming, the opportunity for teaching, and more flexibility in application can make this allocation decision delegation possible. The hope is that this would also lead to a better acceptance of human-robot interaction and a greater sense of joint working on a product. If a worker can decide on their tasks and the tasks of "their" robot, they might be more willing to work together and see the positive opportunity this cooperation offers for their relief and learning opportunities in interaction with technology.

In more standardized production settings, it is possible that this form of delegation is too complicated to implement. Beyond what we found in our experiment, one could assume that participation instead of full delegation would still be an acceptable way to create autonomy and task identity, but that it would have more standardized and controllable allocation decision processes. This would hold for contexts where full flexibility cannot be achieved, be it because of the use of traditional robotics or complex interdependency structures between multiple workers and machines. However, in human-robot interaction in smaller scale production, delegating task allocation to the worker can be a key to unlock the potential of this new form of work.

\subsection{Implications for future research}

With this online experiment, we managed to manipulate the decision influence on task allocation in a fictional setting. As this yielded interesting results, we see the need for further research on the topic of task allocation from a psychological point of view. Research in a more realistic context with real tasks that are executed would be the next step. With this, we will be able to draw better conclusions from real 
human-robot interaction that are better transferrable to practical applications.

It would be of great interest to further investigate the perception of different external decision agents. In this study, we were unable find any differences, but could not determine with absolute certainty the reason for the similarity between the two external agents. We would encourage others to compare different agents and see if their decisions are perceived the same or what characteristics of external agents actually influence a different perception of the decision-making process. It is our intention to also conduct further research on task allocation with different levels of cooperation between a worker and a decision support system, which might yield insights into the specific characteristics (e.g., transparency) that are important for allocation (process) satisfaction and thus for system design in practice.

It would also be very interesting to conduct research with real workers from the manufacturing sector. The results presented in this this study are from a mixed population of very different people who are not and will not necessarily be, affected by human-robot interaction in their daily work. To investigate the implications influence over task allocation can have with people actually affected, and to identify the limits of including them in decisions, it will be necessary to go the next step in redesigning task allocation in human-robot interaction more broadly.

Another vitally important aspect to research is the dynamic of task allocation processes. As quickly changing production demands and changing human needs (e.g., due to increasing strain throughout the work day) require changes in task allocation on a daily basis, concepts like adaptive or adaptable task allocation become more and more important. It is the task of researchers to find out to what degree and how task allocation could and should be adapted to changes in the setting or the person. This needs both basic research on different methods and their objective and subjective outcomes and application-oriented research looking at the presets and boundaries of this dynamic and how it can be implemented in complex production scenarios.

\section{Conclusion}

We showed that the design of allocation processes is immensely important for the experience of human-robot interaction. If the worker is responsible for allocating tasks to themselves and a robot, they experience more autonomy and task identity and are more satisfied with the allocation process, the solution, and the work results than if the task allocation lies with somebody else. If it is another agent deciding on allocation, it does not make a difference whether this agent is an organizational unit or a robot, as long as the decision criteria are appropriate and the agent is trustworthy.
For HRI systems in practice, this shows the important role of consciously designing task allocation processes and not only their solutions.

Acknowledgements The authors would like to thank Dr. Britta Kirchhoff and Dr. Lars Adolph for their ideas and support throughout the conceptualization and execution of the experiment.

Author contributions The theoretical foundation of the study was developed by AT. She conceptualized and implemented the study at the Federal Institute for Occupational Safety and Health, analyzed the data, and was the leading author during the writing process. AK has repeatedly revised the paper in the process and discussed it critically with AT. She actively supervised the writing process, added concrete suggestion on the theoretical concepts, and played a substantial role in designing the structure.

Funding Open Access funding enabled and organized by Projekt DEAL. The research was conducted with resources of the Federal Institute for Occupational Safety and Health. No external funding was utilized.

Data availability The datasets for this manuscript are not publicly available as participants were promised in the data protection declaration that their data would only be accessible for researchers working on the study. Requests to access the datasets should be directed to Alina Tausch at tausch.alina@baua.bund.de.

\section{Compliance with ethical standards}

Conflict of interest The authors declare that the research was conducted in the absence of any commercial or financial relationships that could be construed as a potential conflict of interest.

Open Access This article is licensed under a Creative Commons Attribution 4.0 International License, which permits use, sharing, adaptation, distribution and reproduction in any medium or format, as long as you give appropriate credit to the original author(s) and the source, provide a link to the Creative Commons licence, and indicate if changes were made. The images or other third party material in this article are included in the article's Creative Commons licence, unless indicated otherwise in a credit line to the material. If material is not included in the article's Creative Commons licence and your intended use is not permitted by statutory regulation or exceeds the permitted use, you will need to obtain permission directly from the copyright holder. To view a copy of this licence, visit http://creativecommons.org/licenses/by/4.0/.

\section{References}

Abdallah S, Lesser V (2005) Modeling task allocation using a decision theoretic model. In: Proceedings of 4th international joint conference on autonomous agents and multiagent systems, AAMAS

Alami R, Clodic A, Montreuil V, Sisbot EA, Chatila R (2005) Task planning for human-robot interaction. In: Bailly G, Crowley JL (eds) Proceedings of the 2005 joint conference on Smart objects and ambient intelligence innovative context-aware services usages and technologies, ACM, New York, NY, p 81. https://doi. org/10.1145/1107548.1107574 
Alami R, Clodic A, Montreuil V, Sisbot EA, Chatila R (2006) Toward human-aware robot task planning. In: AAAI spring symposium: to boldly go where no human-robot team has gone before, pp 39-46

Baker M, Yanco HA (2004) Autonomy mode suggestions for improving human-robot interaction. In: 2004 IEEE international conference on systems, man and cybernetics, IEEE, pp 2948-2953

Bakker AB, Demerouti E (2017) Job demands-resources theory: taking stock and looking forward. J Occup Health Psychol 22:273-285. https://doi.org/10.1037/ocp0000056

Brockner J, Wiesenfeld BM (1996) An integrative framework for explaining reactions to decisions: interactive effects of outcomes and procedures. Psychol Bull 120:189

Bucknall T, Thomas S (1996) Critical care nurse satisfaction with levels of involvement in clinical decisions. J Adv Nurs 23:571-577. https://doi.org/10.1111/j.1365-2648.1996.tb00021.x

Cheung JH, Sinclair RR, Shi J, Wang M (2015) Do job demands of Chinese manufacturing employees predict positive or negative outcomes? A test of competing hypotheses. Stress Health 31:432442. https://doi.org/10.1002/smi.2568

Cosenzo K, Chen J, Reinerman-Jones L, Barnes M, Nicholson D (2010) Adaptive automation effects on operator performance during a reconnaissance mission with an unmanned ground vehicle. In: Proceedings of the human factors and ergonomics society annual meeting, vol 25, SAGE Publications, Los Angeles, CA, pp 2135-2139

Dabholkar PA, Sheng X (2012) Consumer participation in using online recommendation agents: effects on satisfaction, trust, and purchase intentions. Serv Ind J 32:1433-1449. https://doi. org/10.1080/02642069.2011.624596

Debernard S, Vanderhaegen F, Millot P (1993) An experimental investigation of dynamic allocation of tasks between air traffic controller and AI systems. In: Strassen HG (ed) Analysis, design and evaluation of man-machine systems 1992. Elsevier, The Hague, pp 95-100

Driscoll JW (1978) Trust and participation in organizational decision making as predictors of satisfaction. Acad Manag J 21:44-56

Fantini P, Pinzone M, Sella F, Taisch M (2017) Collaborative robots and new product introduction: capturing and transferring human expert knowledge to the operators. In: International conference on applied human factors and ergonomics, Springer, pp 259-268

Fitts PM (1951) Human engineering for an effective air-navigation and traffic-control system. National Research Council, Division of Anthropology and Psychology, Committee on Aviation Psychology

Fitzsimons GJ, Greenleaf EA, Lehmann DR (1997) Decision and consumption satisfaction: implications for channel relations. Marketing Studies Center Working Paper Series 313

Flemisch F, Heesen M, Hesse T, Kelsch J, Schieben A, Beller J (2012) Towards a dynamic balance between humans and automation: authority, ability, responsibility and control in shared and cooperative control situations. Cognit Technol Work 14:3-18

Flemisch F, Abbink DA, Itoh M, Pacaux-Lemoine M-P, Weßel G (2019) Cognit Technol Work 21:555-568. https://doi.org/10.1007/ s10111-019-00576

Freedy A, de Visser E, Weltman G, Coeyman N (2007) Measurement of trust in human-robot collaboration. In: Proceedings of the 2007 international conference on collaborative technologies and systems, IEEE, Orlando, FL, pp 106-114

Frey D, Jonas E (2002) Die Theorie der kognizierten Kontrolle [theory of cognized control]. In: Frey D, Martin I (eds) Theorien der Sozialpsychologie, Band III, 3rd edn. Verlag Hans Huber, Bern, pp $13-50$

Gaede C, Ranz F, Echelmeyer W, Hummel V (2018) A study on challenges in the implementation of human-robot collaboration. J Eng Manag Oper 1:29-39
Gombolay MC, Gutierrez RA, Clarke SG, Sturla GF, Shah JA (2015) Decision-making authority, team efficiency and human worker satisfaction in mixed human-robot teams. Auton Robot 39:293312. https://doi.org/10.1007/s10514-015-9457-9

Goodrich MA, Olsen DR, Crandall JW, Palmer TJ (2001) Experiments in adjustable autonomy. In: Proceedings of IJCAI workshop on autonomy, delegation and control: interacting with intelligent agents, Seattle, WA, pp 1624-1629

Habib L, Pacaux-Lemoine M, Millot P (2017) A method for designing levels of automation based on a human-machine cooperation model. IFAC Pap Online 50:1372-1377

Hacker W, Sachse P (2014) Allgemeine Arbeitspsychologie: Psychische Regulation von Tätigkeiten [work psychology: psychological regualtion of tasks]. Hogrefe, Göttingen

Hackman JR, Oldham GR (1975) Development of the job diagnostic survey. J Appl Psychol 60:159

Hackman JR, Oldham GR (1980) Work redesign. Addison-Wesley, Reading

Hancock PA, Billings DR, Schaefer KE, Chen JY, de Visser EJ, Parasuraman R (2011) A meta-analysis of factors affecting trust in human-robot interaction. Hum Factors 53:517-527. https:// doi.org/10.1177/0018720811417254

Hervé M, Pavie X, O'Keeffe M (2000) Valuing people to create value: an innovative approach to leveraging motivation at work. World Scientific, Singapore

Hoc J-M, Lemoine M-P (1998) Cognitive evaluation of humanhuman and human-machine cooperation modes in air traffic control. Int J Aviat Psychol 8:1-32

Hughes R (1998) Considering the vignette technique and its application to a study of drug injecting and HIV risk and safer behaviour. Sociol Health Illn 20:381-400

Inagaki T, Itoh M (2013) Human's overtrust in and overreliance on advanced driver assistance systems: a theoretical framework. Int J Veh Technol 2013:1-8. https://doi.org/10.1155/2013/951762

Jian JY, Bisantz AM, Drury CG (2000) Foundations for an empirically determined scale of trust in automated systems. Int J Cogn Ergon 4:53-71. https://doi.org/10.1207/S15327566IJCE04 01_04

Kaber DB, Riley J (1999) Adaptive automation of a dynamic control task based on secondary task workload measurement. Int J Cogn Ergon 3:169-187

Keen PG (1980) Decision support systems: a research perspective. In: Decision support systems: issues and challenges: proceedings of an international task force meeting, pp 23-44

Lantz PM et al (2005) Satisfaction with surgery outcomes and the decision process in a population-based sample of women with breast cancer. Health Serv Res 40:745-767. https://doi.org/10.11 11/j.1475-6773.2005.00383.x

Locke EA, Schweiger DM, Latham GP (1986) Participation in decision making: when should it be used? Organ Dyn 14:65-79

Michalos G, Spiliotopoulos J, Makris S, Chryssolouris G (2018) A method for planning human robot shared tasks. CIRP J Manuf Sci Technol 22:76-90. https://doi.org/10.1016/j.cirpj.2018.05.003

Moray N, Inagaki T, Itoh M (2000) Adaptive automation, trust, and self-confidence in fault management of time-critical tasks. J Exp Psychol Appl 6:44. https://doi.org/10.1037//0278-7393.6.1.44

Morgeson FP, Humphrey SE (2006) The Work Design Questionnaire (WDQ): developing and validating a comprehensive measure for assessing job design and the nature of work. J Appl Psychol 91:1321-1339

Munzer T, Mollard Y, Lopes M (2017) Impact of robot initiative on human-robot collaboration. Paper presented at the HRI 2017 ACM/IEEE international conference on human-robot interaction, Vienna 
Nikolakis N, Kousi N, Michalos G, Makris S (2018) Dynamic scheduling of shared human-robot manufacturing operations. Proc CIRP 72:9-14. https://doi.org/10.1016/j.procir.2018.04.007

Older MT, Waterson PE, Clegg CW (1997) A critical assessment of task allocation methods and their applicability. Ergon 40:151-171. https://doi.org/10.1080/001401397188279

Onnasch L, Wickens CD, Li H, Manzey D (2014) Human performance consequences of stages and levels of automation: an integrated meta-analysis. Hum Factors 56:476-488. https://doi. org/10.1177/0018720813501549

Parasuraman R, Sheridan TB, Wickens CD (2000) A model for types and levels of human interaction with automation. IEEE Trans Syst Man Cybern Part A Syst Hum 30:286-297. https://doi. org/10.1109/3468.844354

Rajaonah B, Tricot N, Anceaux F, Millot P (2008) The role of intervening variables in driver-ACC cooperation. Int J Hum Comput Stud 66:185-197. https://doi.org/10.1016/j.ijhcs.2007.09.002

Romero D, Noran O, Stahre J, Bernus P, Fast-Berglund A (2015) Towards a human-centred reference architecture for next generation balanced automation systems: human-automation symbiosis. In: IFIP international conference on advances in production management systems, Springer, pp 556-566

Rücker D, Hornfeck R, Paetzold K (2018) Investiagting ergonomics in the context of human-robot collaboration as a sociotechnical system. Paper presented at the advances in human factors in robots and unmanned systems, AHFE 2018

Ryan JC, Banerjee AG, Cummings ML, Roy N (2014) Comparing the performance of expert user heuristics and an integer linear program in aircraft carrier deck operations. IEEE Trans Cybern 44:761-773. https://doi.org/10.1109/tcyb.2013.2271694

Rystedt LW, Devereux J, Sverke M (2007) Comparing and combining the demand-control-support model and the effort reward imbalance model to predict long-term mental strain. Eur J Work Organ Psychol 16:261-278

Schmidt K (1994) Cooperative work and its articulation: requirements for computer support. Trav Hum 57:345-366

Schmidtler J, Knott V, Hölzel C, Bengler K (2015) Human centered assistance applications for the working environment of the future. Occup Ergon 12:83-95

Shaw JC, Wild E, Colquitt JA (2003) To justify or excuse?: a meta-analytic review of the effects of explanations. J Appl Psychol 88:444

Sheridan TB (1984) Supervisory control of remote manipulators, vehicles and dynamic processes: experiments in command and display aiding. In: Rouse WB (ed) Advances in man-machine systems research. JAI Press, New York, pp 49-137

Sims HP, Szilagyi AD, Keller RT (1976) The measurement of job characteristics. Acad Manag J 19:195-212
Slemp GR, Vella-Brodrick DA (2013) Optimising employee mental health: the relationship between intrinsic need satisfaction, job crafting, and employee well-being. J Happiness Stud 15:957-977. https://doi.org/10.1007/s10902-013-9458-3

Spector PE (1986) Perceived control by employees: a meta-analysis of studies concerning autonomy and participation at work. Hum Relat 39:1005-1016

Stanton NA, Salmon PM, Walker GH, Baber C, Jenkins DP (2005) Human factors methods. A practical guide for engineering and design. Ashgate, Farnham

Stegmann S, van Dick R, Ullrich J, Charalambous J, Menzel B, Egold N, Wu TT-C (2010) Der Work Design Questionnaire [the work design questionnaire]. Zeitschrift für Arbeits- und Organisationspsychologie 54:1-28. https://doi.org/10.1026/0932-4089/a0000 02

Thielsch MT, Meeßen SM, Hertel G (2018) Trust and distrust in information systems at the workplace. PeerJ 6:e5483. https://doi. org/10.7717/peerj.5483

Trist E (1981) The evolution of socio-technical systems: a conceptual framework and an action research program. Occas Pap 2 http:// sistemas-humano-computacionais.wdfiles.com/local--files/capit ulo\%3Aredes-sociotecnicas/Evolution_of_socio_technical_syste ms.pdf

Tsarouchi P, Matthaiakis A-S, Makris S, Chryssolouris G (2017) On a human-robot collaboration in an assembly cell. Int $\mathbf{J}$ Comput Integr Manuf 30:580-589. https://doi.org/10.1080/09511 92X.2016.1187297

Van den Steen E (2006) The limits of authority: motivation versus coordination. MIT Sloan Research Paper No 4626-06

Wieland R, Hammes M (2014) Wuppertaler Screening Instrument Psychische Beanspruchung (WSIB) - Beanspruchungsbilanz und Kontrollerleben als Indikatoren für gesunde Arbeit. J Psychol Alltagshandelns 7:30-50

Williams LA, DeSteno D (2008) Pride and perseverance: the motivational role of pride. J Personal Soc Psychol 94:1007-1017. https ://doi.org/10.1037/0022-3514.94.6.1007

Zhang S, Fitzsimons GJ (1999) Choice-process satisfaction: the influence of attribute alignability and option limitation. Organ Behav Hum Decis Process 77:192-214

Publisher's Note Springer Nature remains neutral with regard to jurisdictional claims in published maps and institutional affiliations. 